\title{
Patients from the Oral Oncology Center, UNESP, Araçatuba with an indication for prosthesis
}

\author{
ALDIÉRIS ALVES PESQUEIRA ${ }^{1}$, MARCELO COELHO GOIATO ${ }^{2}$, DANIELA MICHELINE DOS SANTOS ${ }^{2}$, \\ AMÁLIA MORENO ${ }^{2}$, MARCELA FILIÉ HADDAD ${ }^{2}$, PAULA DO PRADO RIBEIRO ${ }^{2}$, \\ LISIANE CRISTINA BANNWART $^{2}$ and GLAUCO ISSAMU MIYAHARA ${ }^{3}$ \\ ${ }^{1}$ University Sagrado Coração (USC), Bauru, SP 17011-160; ${ }^{2}$ Department of Dental Materials and Prosthodontics; \\ ${ }^{3}$ Oral Oncology Center, School of Dentistry, São Paulo State University (UNESP), Araçatuba, SP 16015-050, Brazil
}

Received November 19, 2012; Accepted April 8, 2013

DOI: $10.3892 / \mathrm{mco} .2013 .105$

\begin{abstract}
Head and neck tumors are a major health concern worldwide, due to their high incidence and mortality rates, particularly in developing countries. In Brazil, this type of cancer is commonly diagnosed and studies suggested that it may be the leading cause of mortality in the country. The increase in life expectancy worldwide, as well as environmental and behavioral factors, are related to carcinogenesis. Therefore, an understanding of basic epidemiology and statistical methods is critical, in order to promote early diagnosis and cancer prevention. Cancer patients with an indication for prosthesis were selected from the medical records of the Oral Oncology Center, School of Dentistry, São Paulo State University (UNESP), Araçatuba, between 1991 and 2010. The following variables were recorded: gender, age, type and location of the lesion, radiation dose and dental prosthesis. The majority of the patients were male $(74.15 \%)$ and $>60$ years of age $(53.37 \%)$. Tumors were most commonly located in the floor of the mouth (11.1\%) and squamous cell carcinoma was the most prevalent type $(72.8 \%)$. This study provides the profiles of patients who attended the Oral Oncology Center and the results may aid in the creation of cancer prevention programs.
\end{abstract}

\section{Introduction}

Approximately $10 \%$ of head and neck tumors are malignant and affect multiple sites. Of these, $40 \%$ are located in the oral cavity, $25 \%$ in the larynx, $15 \%$ in the pharynx, $7 \%$ in the salivary glands and $13 \%$ in other locations. Over 8 million new

Correspondence to: Professor Marcelo Coelho Goiato, Department of Dental Materials and Prosthodontics, School of Dentistry, São Paulo State University (UNESP), José Bonifácio 1193, Araçatuba, SP 16015-050, Brazil

E-mail: goiato@foa.unesp.br

Key words: head and neck neoplasms, quality of life, age groups, neoplasms by site, gender identity cases are diagnosed annually, of which 212,000 originate in the oral cavity (1).

Currently, cancer is the second cause of disease-related mortality in Brazil and studies suggested that cancer-related mortality rates may exceed cardiovascular disease-related mortality rates $(2,3)$. Compared to cancer located in other organs, head and neck cancer is relatively common and this anatomical location has the fourth and seventh highest incidence rates among males and females, respectively. In Brazil, $>85 \%$ of the cases are diagnosed at an advanced stage, which reduces survival expectancy and requires a more aggressive therapeutic approach, leading to severe deformities and significantly compromising the quality of life of the patients after treatment (4).

Cancer is a multifactorial disease and is attributed to external or internal factors, which may be interlinked. External factors are associated with the environment, as well as the cultural and social habits of the patient. Internal factors may be systemic, general and genetic. The ability of the body to defend itself from external aggression is another potential factor. These factors may interact in several ways, increasing the likelihood of malignant transformation of normal cells $(2,5)$.

Surgery with or without radiotherapy and chemotherapy is the most common therapeutic approach in the treatment of cancer. The basic principle of cancer surgery involves tumor resection with wide safety margins, which frequently leads to large maxillofacial defects and affects the patient functionally, aesthetically and psychologically. Early diagnosis, combined with currently available treatments (radiotherapy, chemotherapy, surgery and bone marrow transplantation), increases survival rates and improves the quality of life of patients with advanced, incurable cancer $(1,2)$.

Therefore, in addition to tumor resection, it is imperative to rehabilitate patients and preserve their quality of life. Maxillofacial prostheses promote a rapid psychosocial reintegration of patients, are cost-effective, reduce hospitalization time and facilitate the inspection of the affected area $(6,7)$.

The aim of this study was to assess the epidemiological data of cancer patients with an indication for prosthesis who attended the Oral Oncology Center, School of Dentistry, UNESP, Araçatuba, between 1991 and 2010. 


\section{Materials and methods}

The present study was approved by the Human Research Ethics Committee of UNESP, Process FOA/2008-01688.

Patients diagnosed with cancer and an indication for prosthesis were selected from the medical records of the Oral Oncology Center, UNESP, Araçatuba, between 1991 and 2010. Data were reviewed and the variables used in the study were included. A total of 178 medical records were used. Patient consent was obtained from the patient and the patient's family.

Detailed information regarding irradiated patients and radiation dose were not assessed, since a large number of patients had undergone radiotherapy treatment in centers located outside of Araçatuba and that information was not provided by the medical records. Therefore, we registered the responses to this question as 'yes', 'no' or 'treated elsewhere'. Following data collection, the results were analyzed.

\section{Results and Discussion}

Data obtained from the present epidemiological study are presented in Tables I-V.

Gender and age. Table I shows the percentage values regarding patient gender. The majority (74.15\%) of cancer patients were male. Of note, men were more exposed to environmental factors and often presented with backgrounds of smoking and alcohol consumption, which may explain our results. The findings of the present study corroborate those of previous studies which demonstrated a higher incidence rate of head and neck cancer among males $(2,8,9)$. Data obtained from a previous study conducted by Jemal et al (10) demonstrated the same trend in the United States, where 1 in 2 men has a higher risk of cancer development, compared to 1 in 3 women.

A previous study conducted by the National Cancer Institute (NCI) (5) demonstrated that tumors were responsible for $12.73 \%$ of the 946,392 deaths reported in 2000 in Brazil, with 53.97 and $46.01 \%$ of cancer-related deaths occurring in men and women, respectively.

A previous study by Goiato and Fernandes (7) reported that of the 88 cases of laryngeal cancer diagnosed at the Oral Oncology Center in Araçatuba, 80 were men.

According to the NCI (2), oral cancer is the eighth most common cancer among Brazilian men (9,985 estimated cases per year) and the ninth among Brazilian women (3,895 estimated cases per year).

In this study, the age variable was divided into 3 groups (Table I): $0-45,46-60$ and $>60$ years. Subjects $>60$ years of age were the most commonly affected by cancer $(53.37 \%$ of cases). These data are in agreement with the findings of Carvalho et al (8), according to which $75 \%$ of oral cancers were diagnosed in patients $>60$ years of age.

The increasing number of individuals $>60$ years of age has led to an increase of chronic diseases associated with longer exposure to risk factors, such as cardiovascular disease, obesity, diabetes, hypertension and cancer, since only 5-10\% of all cancers are hereditary (individual susceptibility). Approximately $80-90 \%$ of cancers are associated with environmental factors, such as smoking and occupational activities (lung cancer), excessive sun exposure (skin cancer), alcohol
Table I. Patient distribution according to gender and age (absolute no. and percentage of cases).

\begin{tabular}{lc} 
Variable & No. of patients (\%) \\
\hline Gender & \\
Male & $132(74.15)$ \\
Female & $46(25.85)$ \\
Age (years) & \\
$0-45$ & $16(8.98)$ \\
$46-60$ & $67(37.65)$ \\
$>60$ & $95(53.37)$ \\
\hline
\end{tabular}

Table II. Patient distribution according to irradiation and radiation dose (absolute no. and percentage of cases).

\begin{tabular}{lc}
\hline Variable & No. of patients $(\%)$ \\
\hline Irradiated patients & \\
Yes & $116(65.1)$ \\
No & $4(2.2)$ \\
Treated elsewhere & $58(32.7)$ \\
Radiation dose & \\
$>5000$ cGy & $52(44.8)$ \\
$<5000$ cGy & $1(0.9)$ \\
Treated elsewhere & $63(54.3)$ \\
\hline
\end{tabular}

consumption (gastric cancer) and excessive consumption of fatty foods (cancer of the colon and rectum) $(2,5)$. According to Jemal et al (10), $77 \%$ of all cancers are diagnosed in individuals $>55$ years of age.

Irradiated patients and radiation dose. Table II shows the distribution of irradiated patients and the radiation doses. The majority of patients (65.1\%) received radiotherapy treatment, whereas only $2.2 \%$ did not; the remaining $32.7 \%$ had undergone treatment in centers located elsewhere.

Radiotherapy is considered a well-established method of treating or managing malignant head and neck tumors and aims to eradicate tumor cells and preserve the surrounding normal tissue included in the irradiation field (11). Radiotherapy is currently considered the most effective curative cancer treatment next to surgery (1). According to Burnet et al (13), 60\% of patients affected by tumors are likely to be subjected to radiotherapy with curative intent, either alone or in combination with surgery or chemotherapy. Delay in initiation of radiotherapy and treatment interruptions, resulting in prolongation of the total treatment time, are critical factors affecting disease control $(2,13)$.

The oral cavity is prone to radiotherapy complications (12). According to Parliament et al (14), the mandible may receive high doses of radiation, although the maximum dose should not exceed 7,500 Gy.

In this study, $44.8 \%$ of irradiated patients received radiation doses $>5,000 \mathrm{cGy}$. Conventional radiotherapy treatments 
are commonly divided into 5 doses of 180-200 cGy/day, with a total dose of 5,040-7,000 cGy, administered during a time period of 2-8 weeks without interruption, alone or as a complementary treatment, depending on the tumor stage; however, variations may occur (15).

Dentists should familiarize themselves with the currently available protocols for patient management, in order to avoid further complications such as osteoradionecrosis, dryness of the mouth, altered taste and mucositis, which may directly affect the quality of prosthetic rehabilitation of these patients.

Type of cancer. Table III shows the percentage values of different types of cancer. Squamous cell carcinoma had the highest incidence ( $72.8 \%$ of cases), followed by basal cell carcinoma $(4.0 \%)$, melanoma $(2.8 \%)$, cystadenocarcinoma $(2.2 \%)$ and adenocarcinoma (1.7\%).

Among oral cancers, squamous cell carcinoma is the most prevalent type. Its geographical distribution varies in different regions of the world; therefore, it is imperative to determine its pathogenetic as well as its epidemiological profile. Our findings are in accordance with those of Rosa et al (16), who investigated 1,228 cases of oral cancer in the Napoleão Laureano Hospital (João Pessoa, PB, Brazil) over 15 years and reported that squamous cell carcinoma was the predominant lesion $(88.8 \%)$. Furthermore, the literature reveals that $>90 \%$ of head and neck cancers are of the squamous cell type $(9,17)$.

Tumor location. Table IV shows the most prevalent tumor locations among patients of the Oral Oncology Center, UNESP. The floor of the mouth had the highest incidence rate $(11.1 \%)$, followed by the tongue $(10.6 \%)$, palate and oropharynx $(7.3 \%)$.

In a previous study conducted by Sannomiya et al (19) regarding patient distribution according to tumor location in various regions of the oral cavity, the tongue was the anatomical area with the highest incidence rate, with a total of 563 cases ( $54 \%$ of the total sample), followed by the floor of the mouth with 111 cases $(11 \%)$.

According to Sampaio et al (20), of the 236 cases of oral squamous cell carcinoma biopsies registered in the Department of Pathology, UNESP, $26.3 \%$ were located in the floor of the mouth, $25.8 \%$ in the gingiva or alveolar ridge and $17.8 \%$ in the tongue.

Rosa et al (16) also demonstrated a higher prevalence of oral cancer in the tongue and floor of the mouth.

Prostheses. Table V shows the different types of prostheses used by oral cancer patients, among which complete dentures had the highest prevalence $(81.8 \%)$. This result may be due to the fact that the majority of the patients were $>60$ years of age $(53.37 \%)$.

In a retrospective study on the risk of cancer of the upper airway, the use of dentures was not associated with an increased incidence of cancer. However, a history of secondary oral ulcers due to poor denture adjustment and poor oral hygiene (low frequency of toothbrushing) was related to higher cancer risk. Poor oral hygiene may allow for longer contact beween carcinogens (e.g., tobacco or alcohol) and the mucosa (3). However, to the best of our knowledge, no study has confirmed this hypothesis.
Table III. Percentage of different types of cancer.

\begin{tabular}{lc} 
Type of tumor & Percentage $(\%)$ \\
\hline Squamous cell carcinoma & 72.8 \\
Basal cell carcinoma & 4.0 \\
Melanoma & 2.8 \\
Cystadenocarcinoma & 2.2 \\
Adenocarcinoma & 1.7 \\
Other & 16.5 \\
Total & 100 \\
\hline
\end{tabular}

Table IV. Percentages of different tumor locations.

\begin{tabular}{lc}
\hline Location & Percentage $(\%)$ \\
\hline Floor of mouth & 11.1 \\
Tongue & 10.6 \\
Palate & 7.3 \\
Oropharynx & 7.3 \\
Lips & 6.2 \\
Other & 57.5 \\
Total & 100 \\
\hline
\end{tabular}

Table V. Absolute values and percentages of the different types of prosthesis used by oral cancer patients.

\begin{tabular}{lc}
\hline Type of prosthesis & No of patients $(\%)$ \\
\hline Complete dentures & $148(81.78)$ \\
PRD & $18(9.95)$ \\
Fixed partial dentures & $2(1.10)$ \\
Overdentures & $1(0.55)$ \\
Complete dentures + PRD & $4(2.21)$ \\
Obturators dentures & $2(1.10)$ \\
Nasal prosthesis & $1(0.55)$ \\
Tongue prosthesis & $1(0.55)$ \\
Oculopalpebral prosthesis & $4(2.21)$ \\
Total & $181(100.00)$
\end{tabular}

PRD, partial removable dentures.

Dentures must be inspected and well-adjusted in order to avoid mucosal irritation. Patients must be instructed to remove the dentures in case of mucosal ulceration (21).

Based on the results of this study, we concluded that the patients treated at the Oral Oncology Center, School of Medicine, UNESP, Araçatuba, were predominantly men, $>60$ years of age. Several improvements in the information system are required in order to obtain more reliable data and to improve the public health system. This study has provided the profile of patients who attended the Oral Oncology Center and may aid in the creation of cancer prevention programs. 


\section{References}

1. Eckhardt S: Diagnosis, staging, and principles of management. In: Manual of Clinical Oncology. 4th edition. Hossfeld DK, Sherman CD, Love RR and Bosch FX (eds). Springer-Verlag, New York, NY, 1990

2. National Cancer Institute: Estimates of the incidence and cancer mortality in Brazil.http://www.inca.gov.br. Accessed October 23, 2009 (In Portuguese).

3. Velly AM, Franco EL, Schlecht N, et al: Relationship between dental factors and risk of upper aerodigestive tract cancer. Oral Oncol 34: 284-291, 1998.

4. Antunes JL, Toporcov TN and Wünsch-Filho V: The effectiveness of the oral cancer prevention and early diagnosis program in São Paulo, Brazil. Rev Panam Salud Publica 21: 30-36, 2007 (In Portuguese).

5. National Cancer Institute: Coordination of National Cancer Control. PROONCO. The problem of cancer in Brazil. http:// www.inca.gov.br. Accessed September 09, 2009 (In Portuguese).

6. Goiato MC, Fernandes AU, dos Santos DM and Barão VA: Positioning magnets on a multiple/sectional maxillofacial prosthesis. J Contemp Dent Pract 8: 101-117, 2007.

7. Goiato MC and Fernandes AU: Risk factors of laryngeal cancer in patients attended in the Oral Oncology Center of Araçatuba Braz. J Oral Sci 4: 741-744, 2005.

8. Carvalho AL, Singh B, Spiro RH, Kowalski LP and Shah JP: Cancer of the oral cavity: a comparison between institutions in a developing and a developed nation. Head Neck 26: 31-38, 2004.

9. Gervásio OL, Dutra RA, Tartaglia SM, et al: Oral squamous cell carcinoma: a retrospective study of 740 cases in a Brazilian population. Braz Dent J 12: 57-61, 2001.

10. Jemal A, Tiwari RC, Murray T, et al; American Cancer Society: Cancer Statistics. CA Cancer J Clin 54: 8-29, 2004.

11. Pedruzzi PA, Kowalski LP, Nishimoto IN, et al: Analysis of prognostic factors in patients with oropharyngeal squamous cell carcinoma treated with radiotherapy alone or in combination with systemic chemotherapy. Arch Otolaryngol Head Neck Surg 134: 1196-1204, 2008.
12. Da Silva LCF, Paulinelli MN and Meira RA: Assessment of dental and malignancy risk factors in head and neck radiotherapy. Rev Cirur Traumatola BucoMaxiloFac 4: 187-195, 2004 (In Portuguese).

13. Burnet NG, Benson RJ, Williams MV and Peacock JH: Improving cancer outcomes through radiotherapy. Lack of UK radiotherapy resources prejudices cancer outcomes. BMJ 22: 198-199, 2000.

14. Parliament M, Alidrisi M, Munroe M, et al: Implications of radiation dosimetry of the mandible in patients with carcinomas of the oral cavity and nasopharynx treated with intensity modulated radiation therapy. Int J Oral Maxillofac Surg 34: 114-121, 2005.

15. Bese NS, Hendry $J$ and Jeremic B: Effects of prolongation of overall treatment time due to unplanned interruptions during radiotherapy of different tumor sites and practical methods for compensation. Int J Radiat Oncol Biol Phys 68: 654-661, 2007.

16. Rosa MRD, Falcão AV and Sampaio GS: Epidemiology of oral cancer in Paraiba: Brazil (1978-1993). Rev Bras Ciênc Saúde 1: 17-22, 1997 (In Portuguese).

17. Casiglia $J$ and Woo SB: A comprehensive review of oral cancer. Gen Dent 49: 72-82, 2001.

18. Oliver AJ, Helfrick JF and Gard D: Primary oral squamous cell carcinoma: a review of 92 cases. J Oral Maxillofac Surg 54: 949-954, 1996.

19. Sannomiya EK, Medici Filho E, Moraes LC, et al: Evaluation of radiotherapy and chemotherapy treatment in patients of oral squamous cell carcinoma. Rev Odontol UNESP 32: 119-124, 2003 (In Portuguese).

20. Sampaio MCC, Birman EG and Novelli MD: Carcinoma espinocelular de boca - estudo clínico de 236 casos. Ars Cvrandi Birman Odont 8: 69-76, 1981 (In Portuguese).

21. Declerck D, Vinckier F, Matterne and Van Limbergen E: Dental care in patients undergoing radiotherapy of the head and neck. Rev Belge Med Dent 50: 47-67, 1995 (In French). 